\title{
BJMHR
}

British Journal of Medical and Health Research

Journal home page: www.bjmhr.com

\section{Effect of Polypharmacy in Geriatrics Population -A Pharmacovigilance Study}

\author{
Athira SR ${ }^{1}$, Arya KS ${ }^{1}$, Kiron $S^{1 *}$, Balakrishnan Valliyott ${ }^{2}$ \\ 1.Pariyaram medical college, Kannur(DIST), Kerala \\ 2.Dept. of Medicine, Pariyaram Medical College, Kannur, Kerala
}

\section{ABSTRACT}

As per the census of 2001 geriatric population accounts for $7.4 \%$ of total population which increases to $8.2 \%$ by 2011 and likely to be $10.7 \%$ by 2021. Aging is associated with decreased renal and hepatic clearance of drugs and about one third of the elderly patients are hospitalized due to adverse drug reactions and leading cause were polypharmacy and comorbidities. The objective was to identify and spontaneous reporting of ADR observed in geriatric patients and to assess the influence of polypharmacy and co-morbidities causing ADRs. A six months prospective study was conducted in geriatric patient of medicine department, Pariyaram Medical College, Kannur (DIST) after obtaining the Ethical Approval. Study was based on the identification, reporting and assessment of ADR according to the inclusion and exclusion criteria. Any reaction noted will be brought into the notice of the physician and filled the notification form. These reports were coordinated during ward rounds and pharmacy practice activities. Informed consent shall be taken from the patient for suspected ADR before documentation. Data of each patient was collected using structured data collection form. The results were statistically analyzed using SPSS version 21. A total of 203 geriatric patients, 148 patients were monitored during the study period. In 61 ADR reported; $62.29 \%$ were female and $37.70 \%$ male. Antibiotics were the most ADR causing class of drug and the gastrointestinal system(27.86\%) was mostly affected. Majority of patient had comorbidity condition of diabetes $(70.76 \%$ ) and $93.6 \%$ of study population consumes more than 6 drugs. By applying Spearson',s rho stastical method age was strongly correlated with polypharmacy $(\mathrm{p}=0.000)$. Causality assessment according to Naranjos scale observed that $18.03 \%$ of ADRs were definite , $52 \%$ possible and probable comprises $29.5 \%$. Polypharmacy comes with an increased risk for negative health outcomes such as higher healthcare costs, ADEs, drug-interactions, medication non-adherence, decreased functional status and geriatric syndromes. Another reason of ADR is comorbid condition. The available data suggest that a scope of closer pharmacovigilance studies are much needed in the older age group due to co morbidity and polypharmacy which can cause ADR that are preventable.

Keywords: Geriatric, Polypharmacy, Co-morbidities, Pharmacovigilance. 


\section{INTRODUCTION}

Drug is a double edged sword where the single active chemical entity used for diagnosis, prevention, treatment, cure of a disease but the adverse drug reactions (ADRs) represent a common problem during drug treatment (K.D Tripathi; 2013). Predisposing factors such as polypharmacy, multiple intercurrent diseases and physiologic difference between men and women can increase the risk of ADR. (Parthasarathy G,Nyfort Hansen K and Nahata $\mathrm{M}$;2008).The cost of managing and the inconvenience caused by the ADR to both the patient and caregiver will be exponentially high. For inpatients, it may increase the length of stay which causes additional risk of developing newer ones.(Kiran Nagaraju et.al;2014)

Ageing is a biological reality, there will be progressive deterioration of all body functions which may make the individual difficult to carry out their day to day activities. Most of the developed countries had accepted the chronological 65 or above 65 as elderly or older people. They are often referred as Geriatric population. ${ }^{4}$ (Alessandro Nobili,Silvio Garattini, Pier Mannuccio Mannucci;2011). The important way to identify the particular ADR is to obtain the previous history of such ADRs occurred and it should be documented (Rima Shah et.al 2012; Oxford University Press;2009). Since the pharmacokinetic and pharmacologic profile will be altered in the older people, dose titration, therapeutic drug monitoring should be always done carefully(P.A Roultedge, M.S O Mahony and K W Woodhouse;2004)

By the last two three decades a large number of toxic materials had been introduced in the marketed as drugs. However, the ADR reporting in India is still in its infancy. It may be due to the lack of awareness or lack of interest of health care professionals in reporting Here the pharmacist can take an active role in reporting through clinical meetings they can encourage other health teams in reporting. (Alessandro Nobili,SilvioGarattini,Pier Mannuccio Mannucci;2011). The National Pharmacovigilance Programme established in January 2005 was to be overseen by the National Pharmacovigilance Advisory Committee based on the Central Drug Standard Control Organization (CDSCO), New Delhi. The present study was initial pace in initiating the ADR reporting and documenting in geriatric populations in northern part of Kerala to improve the quality of life

\section{MATERIALS AND METHOD}

\section{Study Site:}

The study was conducted at inpatient and outpatient Medical departments of Pariyaram Medical College, Kannur (DIST). It is a 1200 bedded tertiary care teaching hospital. The study was performed based on the identification, reporting and assessment of ADR in geriatric populations from multiple departments. These reports were coordinated during ward rounds and pharmacy practice activities. The Health Care practitioners participate in 
Pharmacovigilance program and employ a system of spontaneous reporting, which was the form of reporting used in this study.

\section{Study Design:}

It was a prospective, observational study for identifying and reporting the ADR at a tertiary care teaching hospital at Kannur for a time period of six months. Patient above 60 years were included in the study. Those patients who were excluded from the study are age below 60 years, with intentional or accidental poisoning, who developed an ADR during transfusion of blood or blood products, patients with drug abuse and with accidental or emergency cases Medical staff, medical post graduates, nursing staff and patients were educated and encouraged to report ADRs by creating awareness through brief presentations and conducting clinical meetings. ADR notification forms were kept in the all nursing stations of medicine ward and ICU. Patients were assessed for ADRs during the study period. In suspected cases, patient's past medical history and medication history were collected. Patients were monitored daily throughout their hospital stay, and some their medical records were reviewed daily. Any reaction noted will be brought into the notice of the physician, who if convinced enough of the drug cause of reaction filled the notification form Informed consent shall be taken from the patient for suspected ADR before documentation. Data of each patient was collected using structured data collection form. For details of each patient, an ADR form was completed with regard to patient age, sex, diagnosis, prescribed medications, daily doses, treatment, indications for each drug, adverse drug reaction occurred, laboratory investigation reports and history of the patient. Any drug treatment and/or supportive therapy given for the management of the reactions were also be noted. The reported ADRs were classified according to Rawlins and Thompson classification and causality assessment was done with Naranjo's scale

\section{Evaluation of data:}

\section{Patient characteristics}

Patient's age and gender were considered in the evaluation. The study comprises the patients of either sex above 60 years of age who developed an ADR admitted in medicine ward and medical ICU. Study populations were classified according to the US classification of Geriatrics into young old, middle old and old-old. The young old include the age between 6574 , middle old includes 75-84 and old-old are those who were 85 and above.

\section{Reaction Characteristics}

ADR were classified according to Rawlins and Thompson classification, as type A or type B. Type A include the exacerbation of pharmacological action and Type B reactions represent allergic and idiosyncratic reactions that are independent of drug pharmacology. It was also 
classified into Mild, Moderate and Severe reactions based on the classification system of Hartwig. et al. Mild reactions were those that are self -limiting, resolved over time without treatment, and did not extend a patient's hospital stay. Moderate ADRs were definite as those that required therapeutic intervention and prolongation of hospital stay by one day but that are resolved within 24 hours due to a change in drug therapy or the administration of specific therapy. Severe ADRs were threatened patients' lives causes' disability etc. ADRs were also classified based on the organ system affected by the ADR.

\section{Drug characteristics}

The drugs involved in the ADR were categorized into various drug classes according to their therapeutic classification.

\section{Multiple or Inter current diseases}

Patients who already have multiple or inter current disease can also predispose to the development of ADR. They were reviewed daily and their medication and laboratory data were closely monitored.

\section{Causality Assessment}

Causality assessment of ADR was carried out using Naranjo's scale which categorizes the causality relationship into definite, probable, possible and unlikely. By answering 10 questions like timing between administration of the drug and development of the reaction? Does the reaction worsen with repeated or increased dosing? Does the reaction decrease in intensity when the dose of the drug is reduced or discontinued? Has the patient previously been exposed to the drug, in cases of allergic reaction? Is the reaction known to occur with long-term use of the medication? Did symptoms appear or worsen when a drug was discontinued? Answering such questions can help the pharmacist determine causality about the ADR and assigning a numeric score to each answer, the ADR probability classification can be determined. If the score was about 9 it is "definite" and if it was between 5-8 it was "probable". If the score was between1-4 it was "possible" and when it was less than 0 it comes under the category of" unlikely". ${ }^{3}$ Severity of ADR was graded as per scale developed by Hartwig. et al.

\section{Stastical Analyses:}

The data were entered and coded, and descriptive statistics were calculated for all survey items. All statistical analysis were conducted using SPSS version. The result are expressed as percentage and presented in graphs. In addition, the associations between the variables were determined by performing Spearman's rho correlation, Chi square test and independent ' $t$ ' value test. A p value<0.05 was considered significant in all analyses.

\section{Ethics and Consent:}


The study was approved by the Institutional Human Ethic Committee of Academy of Pharmaceutical Sciences, Pariyaram Medical College filed under NO:A1/1839/2016/APSC/IEC dated on 03/2016. Permission to conduct the study was obtained from the Medical Superintendent of Pariyaram Medical College Hospital.

\section{RESULT AND DISCUSSION}

A total of 203 patients included during the six-month study period .Data were collected from all the Medical departments and also counting from Sahakarana Hrudayalaya. In that 123 patients were monitored, out of which 61 ADRs were identified and documented.

\section{Patient characteristics:}

Among the stated our study populations, mean age was 75.65(SD \pm 8.22$), 50.4 \%$ (62 patients) was in the Young old category (Figure-1, 33.3\% (41 patients)in the middle old category and the least occupies in old old category $16.2 \%$ (20 patients).

Most of the developed countries had accepted the chronological 65 or above 65 as elderly or older people According to the US Geriatric classification, the study population was divided into 3 categories young old, middle old and old-old. The young old include the age between 65-74 middle old includes 75-84 and old-old are those who were 85 and above. The mean age of study population was $74.23 \pm 7.318$ and the age category was significantly correlated with the system affected by adverse reactions and polypharmacy ( $\mathrm{p}$ value $=0.00$ ). The individual enters to the last phase of life cycle, there will be progressive deterioration of all body functions. Similar to our reports, another three studies from India carried out also enlightened that majority of the geriatrics patients fell within the age range of 65 to 74 years by (Rima Shah et al;2012 and V. Kalaiselvan et al;2014, Maheshkumar Pauldurai et al;2015)

Amid the documented adverse drug reactions, 62.29\% (38 ADR) were reported from the female patients ie, more than half of the adverse drug reactions. Only $37.70 \%$ (23 ADR) were reported from the male ones. Physiologic difference between men and women play an important role in disease prevalence, drug activity including pharmacokinetic and pharmacodynamic parameters and treatment outcomes. Women in comparison with men have lower body weight and organ size but have a more body fat and a different gastric motility. This physiologic difference made women more prone to adverse drug reactions. (Parthasarathy G ,Nyfort Hansen K and Nahata M;2008) Similar to our data studies found out that in most of the adverse drug reaction were reported in female patients than the male. (Conforti et al;2012 and Kiran Nagaraju et al;2015,Jha et al;2007and Tejal et al;2003).But contrasting to the above results, studies were reported from the male than the female patients.(V. Kalaiselvan et al;2014 Asawari Raut et.al;2016 and Bilal Ahmed et al;2014)

These dissimilarities may be due to variation in study populations in different studies. 


\section{In Patient-Outpatient Category:}

In this study, inpatients developed more ADRs than the out-patients. Of the total 61 reactions reported, $80.3 \%$ (49) were reported by the inpatients and only $19.6 \%$ (12) from outpatients. Among the adverse reactions reported most of them were observed with the in patients. It may be due to more severity of diseases, multiple drugs and also patients were at an increased chance of nosocomal infections during the hospital stay. Another work also concludes the same result (Rima Shah et.al; 2012). The effective pharmacovigilance programs in hospital setup possibly will influence these results.

Among 61 reactions reported 70.49\% (43) were reported by the medical staffs which include practicing physicians, nursing staffs and medical students .Only 19.67\% (12) were reported by the PharmD students during the daily ward round and the least from patients $9.83 \%(6)$ through a successful patient interview. The different studies concluded that ADR were reported maximum by nursing and Medical staff (Rogie Royce Carandang et al; 2015), Pharmacist (Annemie Somers.et.al; 2003)

\section{Reaction Characteristics:}

In the study, ADRs were characterized based on Rawlins and Thompson classification. Out of 61 ADR reported $31.47 \%$ (19) were type A which include the exacerbation of pharmacological action and $68.85 \%$ (42) were Type B reactions which represent allergic and idiosyncratic reactions that are independent of drug pharmacology (Figure- 3). Be on familiar terms, Type A reactions are just exacerbation of pharmacological actions and most of the adverse reactions observed are precipitated likewise only. In our study patients had developed insulin induced hypoglycemia, number of patients had frusemide induced electrolyte imbalance like hypokalemia, hyponatremia and hypomagnesemia and propanalol induced hypotension was also among them. Type B reactions are occurred due to idiopathic causes. In this study most of the adverse reactions were type B category. The statistical analysis shows the type of ADR was significantly correlated with the ADR occurred in different physiological system $(\mathrm{p}$ value $=0.005)$

Study from India and a study from Northern Brazil were parallel to our study and they also observed that hypersensitivity type of reactions have the major association among ADRs( Mohammed Abzur Ghufran et al;2016 and Marcia Germana Alves de Araujo Lobo et al;2013).Contradicting to the above facts another two studies with their observation stats that majority of ADR reported were classified as type A reactions (Hanlon $\mathrm{J}$ T et al;2001and Marie-Laure Laroche et al;2007). All data contrasts may be due to variations in study setup. assessment of adr severity: 


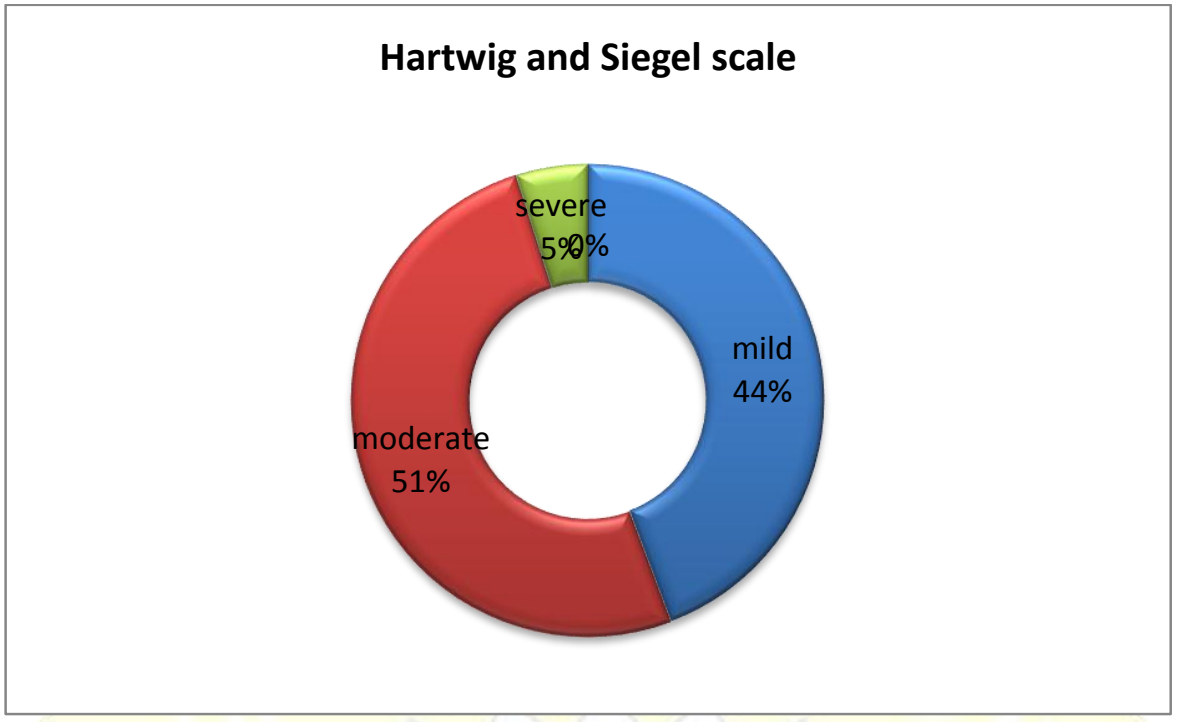

Figure 2: Severity Assessment Based On Hartwig And Siegel Scale

According to the classification of Hartwig and Siegel scale the adverse drug reactions were classified into mild, moderate and severe. In the present study, out of 61 reactions observed $44.26 \%(27)$ were mild and $50.81 \%$ (31) as moderate which extent the hospital stay of the patient (Figure- 4$)$. Only $3(4.91 \%)$ cases were reported as severe.

Among the 27 milder conditions, $77.77 \%(21)$ were classified as level-1 reactions and $28.57 \%$ (6) included in level-2 reactions(Figure- 6). In the moderate 31 cases, more than half of the total reaction $(61.29 \%$ (19)) were include in the moderate level- 3 classifications and the rest $12(38.70 \%)$ were assessed as moderate level- 4.Only 3 reaction were founded to be included in the severe class and each of them were included in level 56 and 7 of severe class. Mild reactions were those that are self -limiting, resolved over time without treatment, and did not extend a patient's hospital stay. Moderate ADRs were defined as those that required therapeutic intervention and prolongation of hospital stay by one day but that are resolved within 24 hours due to a change in drug therapy or the administration of specific therapy. Severe ADRs were threatened patients lives causes disability etc. However, the limitation of the scale is that, in a given case of ADR the level of severity can be assigned only at the final outcome. This reduces the use of scale to an academic exercise only.

There was a number of studies coincides with our result. The studies done in India in rural hospitals were found that majority of reactions were moderate (Jamuna Rani et al;2011, Tejal et al;2003 and Asawari Raut et al;2016,Mohammed Abuzar Ghufran et al;2016). Contradicting to this work based on increase risk of ADR in ambulatory elders revealed that occurrence of ADR was more common in serious than in milder ones (Michael A. Steinman et al; 2011). Our findings could be comparable with studies performed by prior researchers.

\section{Organ System Affected By The Adr:}




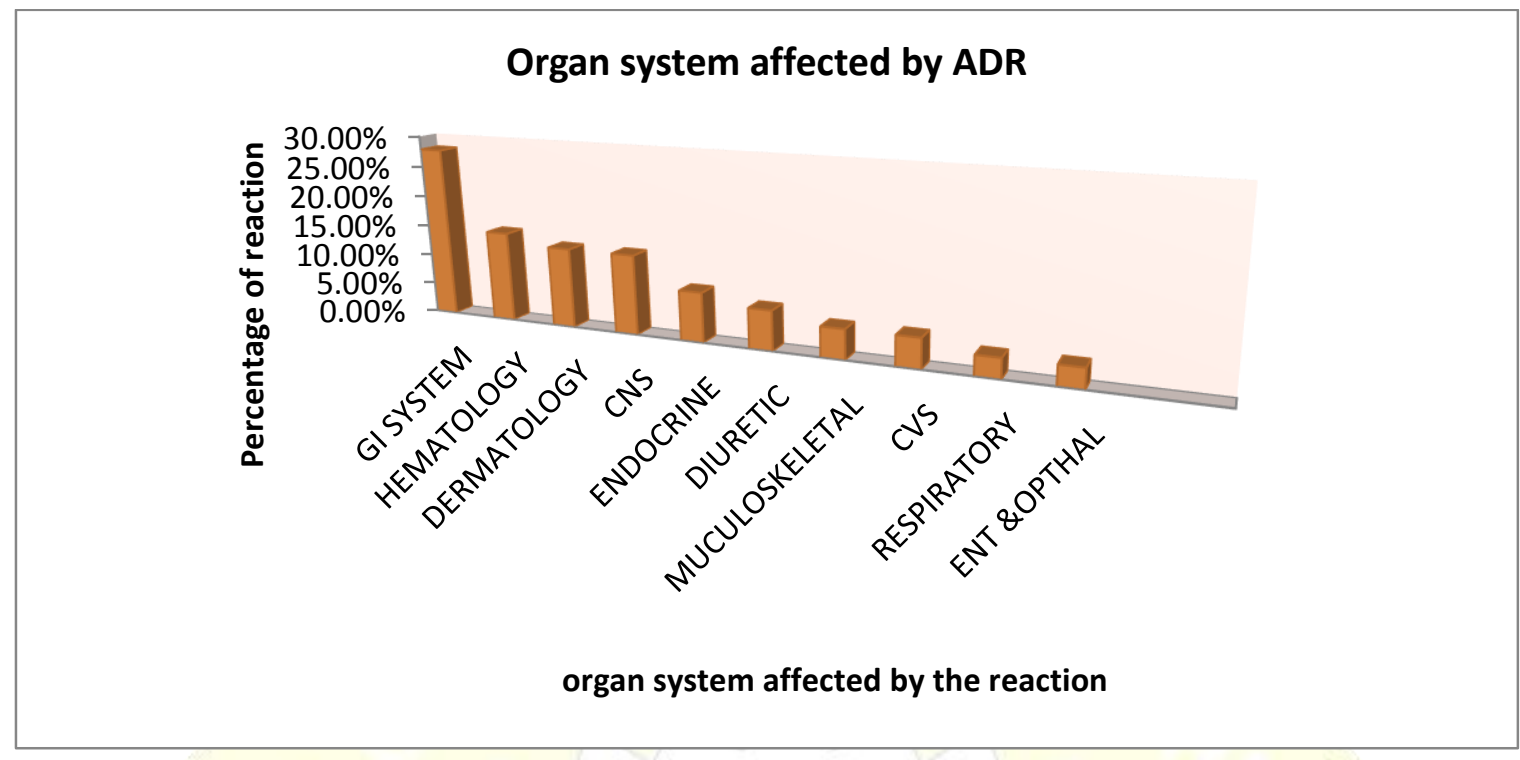

Figure 3: Percentage Of Organ System In Adr Reporting

In the study, we found that several organ systems were affected by medications. Adverse reaction mostly affected was the gastrointestinal system $17(27.86 \%)$ followed by hematology $9(14.75 \%)$ then dermatology and Central nervous system, Endocrine system, Diuretic system, Musculoskeletal and cardiovascular system. The last by respiratory system, ENT and opthal (Figure-5). Another studies were also coinciding with our result that majority of reported adverse drug events had affected gastrointestinal system(Maheshkumar Pauldurai et al;2015 and Rima Shah etal;2012 ,Gray S L et al;1999).Counter to this studies highest frequency of adverse reactions were occurred in the dermatological system manifesting as formication, skin rashes, flushing and dried skin. (Marcia Germana Alves de Araujo Lobo et al;2013 and R.Arulmani et al;2007)

\section{Drug characteristics:}

The details were given in Table- 4.Due to deteriorating physiological conditions most of them were consumed more than two types of drugs especially antihypertensive and hypoglycemic agents.

Table- 1: - List of Drug Induced ADR

\begin{tabular}{ll}
\hline Disease & Number \\
\hline Antibiotics & 8 \\
Antiplatelet & 7 \\
Anticoagulant & 7 \\
Diuretics & 6 \\
Antihypertensive & 5 \\
Antidabetics & 5 \\
Antiepileptics & 4 \\
Antipsychotics & 2 \\
Bronchodilators & 2 \\
Antimalarials & 2 \\
Others & 13 \\
\hline
\end{tabular}


Out of 61 reported ADRs, 8 patients were developed ADR with antibiotics, followed by anticoagulant, antiplatelet, antihypertensive, anti-diabetics, Anti epileptics, bronchodilators, antimalarials, antipsychotics and the rest includes anticholinergics, antivirals, cardiac glycosides, hypolipidemics, antiemetic, laxatives nutrient supplements which all together forms others (13). A study from UK by clashes with the above results, showed that most frequently implicated drug groups causing ADRs in elderly were loop diuretics, opioids, steroids, anticoagulants and antimicrobials (Davies EC et al;2009). The other work correspondingly revealed that cerebral vasodilators (36.4\%) were the drugs most frequently used developed ADRs (Marie-Laure Laroche et al;2007). Another two studies from India done by found that cardiovascular drugs and antimicrobials were the commonest drugs leading to ADR in elderly which was similar in our study also (Sharma H et al; 2007 and Rima Shah et al; 2012).

\section{Polypharmacy:}

Likewise, study assesses the influence of polypharmacy in progress of ADRs. Among the 61 adverse reactions, $54 \%$ (33) patients were prescribed with 6-10 drugs and $34.4 \%$ (21) were prescribed with $11-15$ drugs. Only $6.5 \%$ (4) were consuming with minimum number of drugs ie.,0-5 and even 4.9\% (3) were prescribed with 16-20 drugs (Figure- 8).

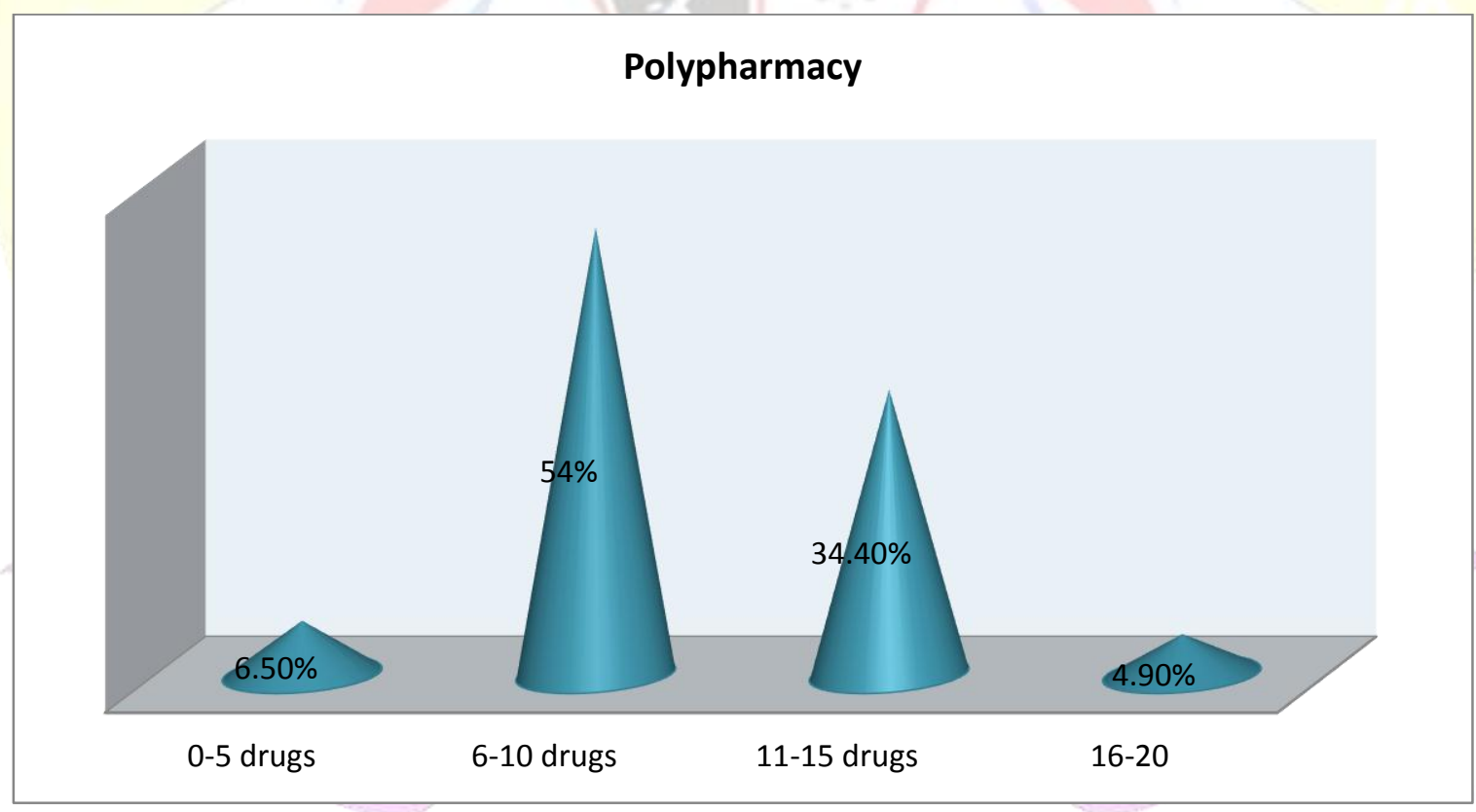

Figure 4: Percentage Of Number Of Drug Administrated

Polypharmacy which means the use of multiple medication typically 5 or more. It is often used when prescribing more medication or inappropriate medication for a clinical condition. As people get older they were prescribed with multiple drug therapy are more prone to develop an ADR either due to alteration of drug effect through an interaction mechanism or by an synergistic effect. The amount of risk associated with multiple drug therapy increases 
in direct proportion to the number of drug administrated. By applying statistics Spearson's rho method age was strongly correlated with polypharmacy $(\mathrm{p}=0.000)$. According to one of the study majority of their study population conceive 6-10 drugs (Kiran Nagaraju et al;2015, Marcia Germana Alves de Araujo et al;2013). But another study in Nigeria (51.8\%) patients had 3-4 drugs in their prescription (Fadare et al;2013). Another one explain the occurrence of polypharmacy was $70 \%$ and the incidence of ADRs was $10.5 \%$ among the study cohort(Bilal Ahmed et al;2014).

\section{Multiple or Intercurrent Diseases:}

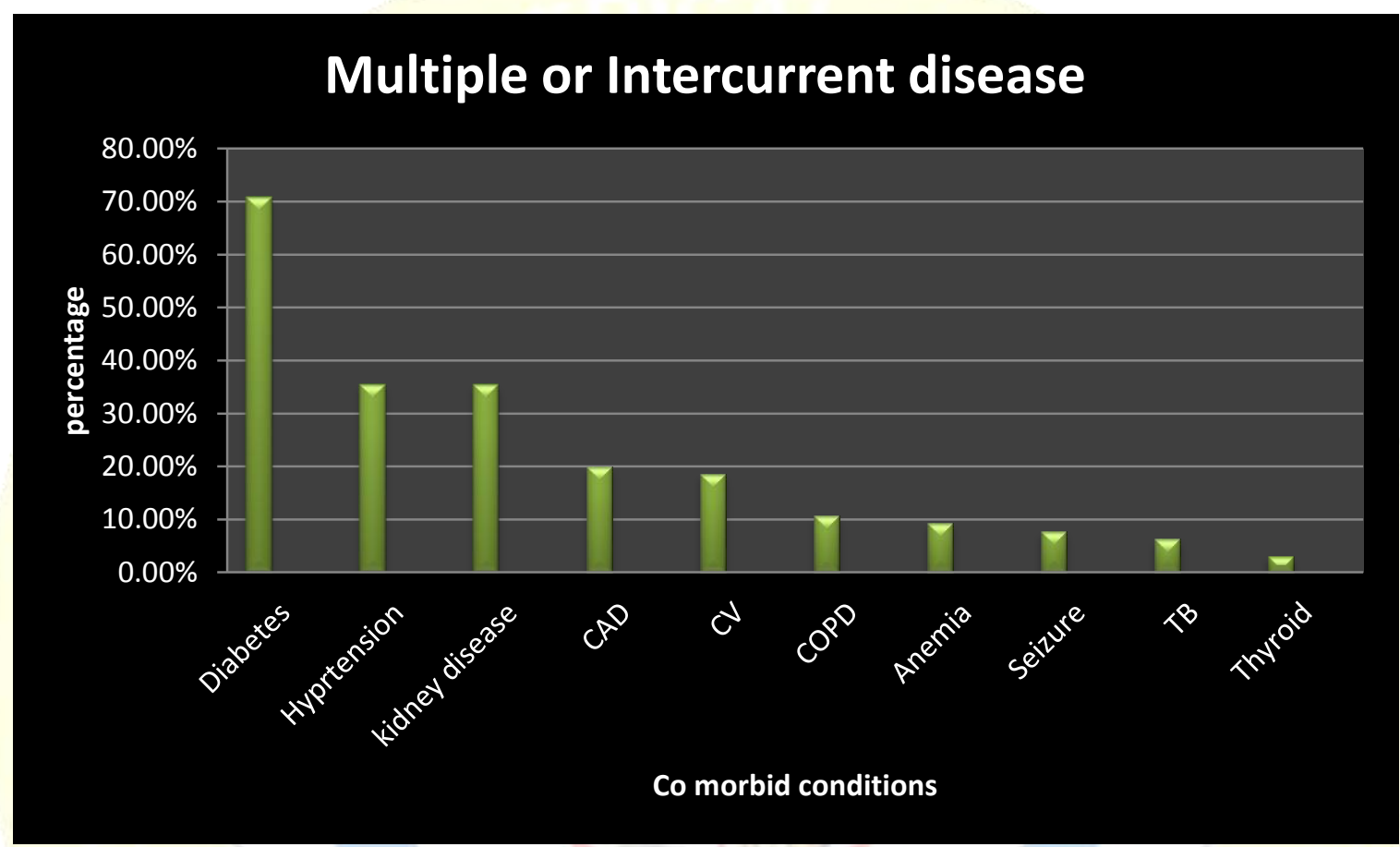

Figure 5: Percentage Of Co Morbid Condition In Study Population

Out of 123 patients, $70.76 \%$ population had diabetes, tailed by $35.38 \%$ had Hypertension and kidney disease, $20 \%$ coronary artery disease, $18.46 \%$ cerebrovascular disease, $10.76 \%$ COPD, 9.23\% anemia, $7.69 \%$ seizure, $6.23 \% \mathrm{~TB}$ and $3.07 \%$ (Figure-7) had thyroid problem.

Aging will not come alone, it is accompanied by chronic disease, co morbidities, disabilities etc. An Indian study concluded that most commonly found chronic conditions were hypertension (Kiron Nagaraju et al;2015). Another one carried out the study in Nepal found that most common diagnoses were respiratory disease and cardiovascular disease (Lohani et al;2006, Pauldurai et.al;2015). The statistical correlation was performed between age and Comorbidities shows (Table-.2) the significant correlation with DM, Hypertension, Kidney Disease, CAD, CVD and Thyroid.

Table2 :Comorbidity Vs Independent ' $t$ ' value

\begin{tabular}{ll}
\hline Comorbidity & Independent 't' value \\
\hline Diabetic Mellitus & 0.000 \\
Hypertension & 0.000 \\
\hline
\end{tabular}




\begin{tabular}{ll}
\hline Kidney disease & 0.002 \\
Coronary artery disease & 0.000 \\
Cardiovascular disease & 0.000 \\
COPD & 0.117 \\
Anemia & 0.235 \\
Seizure & 0.264 \\
Thyroid & 0.004 \\
Tuberculosis & 0.359 \\
\hline
\end{tabular}

\section{Causality Assessment:}

\section{Naranjo's causality assassement}

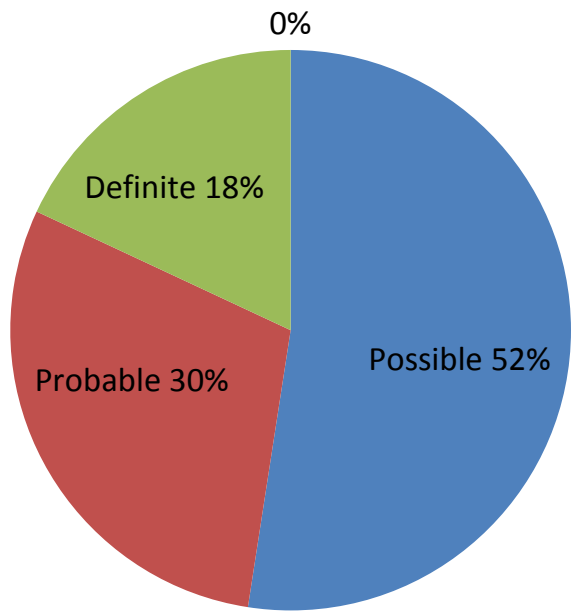

Possible

Probable

Definite

Unlikely

\section{Figure 6:Causality Assessment Based On Naranjo Scale}

Causality assessment of ADR was carried out using Naranjo's scale which categorizes the causality relationship into definite, probable, possible, unlikely. Among the 61 reported ADRs only $18.03 \%$ (11 ADR)comprises definite(Figure-10). Much more increase number had been observed in case of probable $(29.5 \%(18))$ and the greatest ADR was with possible (52\% (32)) category. Similar to the above datum majority of the reaction were classified in the "Possible" category (Mohammed Abuzar Ghufran et al;2016 Asawari Raut et al;2016). But a study from South India, most of the ADRs were categorized as "Probable" and no ADR was found in "Unlikely" class (Palanisamy et al;2009).

\section{CONCLUSION}

A total of 203 geriatric patients, 148 patients were monitored during the study period. In 61 ADR reported; $62.29 \%$ were female and $37.70 \%$ male. Antibiotics were the most ADR causing class of drug and the gastrointestinal system(27.86\%) was mostly affected. Majority of patient had comorbidity condition of diabetes $(70.76 \%)$ and $93.6 \%$ of study population consumes more than 6 drugs. Causality assessment according to Naranjos scale observed that 
$18.03 \%$ of ADRs were definite, $52 \%$ possible and probable comprises $29.5 \%$. Physicians and post graduates $(70.49 \%)$ were reported more number of ADRs during hospital stay.

Age related changes in drug disposition and pharmacodynamics response have significant clinical implications; increased use of a number of medication raises the risk that medicine related problem may occur. Polypharmacy comes with an increased risk for negative health outcomes such as higher healthcare costs, ADEs, drug-interactions, medication nonadherence, decreased functional status and geriatric syndromes. Another reason of ADR is comorbid condition. Majority of ADR causing admission or occurring in hospital are type A reaction. The first step to be taken is the reduction in the number of drugs administered is the most beneficial way to enhance the quality of the treatments given. Polymedication reduction in the elderly should be a constant preoccupation of physicians for a direct beneficial effect, allowing a decrease of the prevalence of drug-drug interactions and for easing the burden on healthcare costs. The available data suggest that a scope of closer pharmacovigilance studies are much needed in the older age group due to co morbidity, which can cause ADR that are preventable.

\section{LIMITATION OF THE STUDY}

- Six months duration we are not able incorporate all the geriatric populations admitted in our hospital.

- Only 148 patients were able to monitor due to time lag.

- Due to insufficient resources we are unable to collect the data from all the departments.

- Due to reduced sample size we are not able to done the proper statistical analysis.

- We may not have identified all medicinal products which produce adverse drug reaction in our hospital departments due to shortage of time.

\section{FUTURE RECOMMENDATION}

- In future studies elderly patients need more focus to detect more ADR..

- More efforts should be made towards strengthening the drug monitoring systems in our hospitals in association with other healthcare professionals.

- Pharmacy practice department should encourage health professions and patients in reporting suspected adverse drug reactions and they motivated through conducting CMEs.

- Study will continue to the next academic year for including more number of samples in collaboration with Pharmacovigilance of our hospital for better patient care.

- Incorporation of advanced technology and mobile applications for improved ADR reporting strategy 
Conflicts of interest statement: All authors have declared no potential conflicts of interest regarding this study.

Acknowledgments: The authors thank to the all students and, teaching and non teaching staff of Academy of Medical Sciences Pariyaram , Kannur, Kerala

Financial disclosure: this work was not financially supported by any means

\section{REFERENCE}

1. K D Tripathi. Essential of medical pharmacology. JAYPEE brothers medical publisher (P) LTD: 2013;7:2-5.

2. Parthasarathy G, Nyfort Hansen K and Nahata M. A textbook of clinical pharmacy practice -essential concept and skills. Orient blackswan .2008; 28: 85-90.

3. Kiran Nagaraju et.al. Pharmacovigilance in Geriatrics. International Research Journal of Pharmacy. 2014; 5(12): 876-878.

4. Alessandro Nobili, Silvio Garattini, Pier Mannuccio Mannucci . Multiple diseases and polypharmacy in the elderly: challenges for the internist of the third millennium. Journal of Comorbidity 2011; $1: 28-44$.

5. Rima Shah et al. A Profile of Adverse Drug Reactions with risk factors among Geriatric Patients in a tertiary care teaching rural hospital in India. National Journal of Physiology, Pharmacy \& Pharmacology. 2012;2 ( 2) : 113 - 122.

6. The Author 2009. Published by Oxford University Press on behalf of the British Geriatrics Society. Adverse drug reactions in elderly: challenges in identification and improving preventative strategies.

7. P.A. Roultedge,M S O Mahony and K W Woodhouse : Adverse Drug Reaction in Elderly Patient.Br J Clin Pharmacol 2004; 57(2) :121-126

8. Asawari Raut et al.Monitoring of Adverse Drug Reaction In Elderly Patient In An

9. Annemie Somers et al.Reporting Adverse Drug Reaction on a Geriatric ward :A Pilo Project.European Journal Of Clinical Pharmacology 2003;58(10):707-714

10. Hanlon JT, Maher RL, Lindblad CI, et al. Comparison of methods for detecting potential adverse drug events in frail elderly inpatients and outpatients. Am J Health Syst Pharm. 2001; 58(17):1622-1626.

11. Gray SL, Mahoney JE, Blough DK. Adverse drug events in elderly patients receiving home health services following hospital discharge. Ann Pharmacother. 1999; 33(11):1147-1153.

12. Marcia Germana Alves de Araujo Lobo et al..Adverse drug reaction monitoring: support for pharmacovigilance at a teritiary care hospital in Northern Brazil .BMC Pharmacology and Toxicology .2013: 3-7 
13. V Kalaiselvan et al.Spontaneous Reporting of Adverse Drug Reactions in Geriatric patients in India. National Journal of Physiology, Pharmacy \& Pharmacology .2014; $4(3): 225-228$

14. Maheshkumar Pauldurai, Dhanapal Kannaaiyan and Ramakrishna Rao. Adverse drug reaction monitoring in geriatric patients of rural teaching hospital .Der Pharmacia Lettre. 2015; 7 (12):187-193

15. Conforti A, Costantini D, Zanetti F, et al. Adverse drug reactions in older patients: an Italian observational prospective hospital study. Drug Healthc Patient Saf . 2012; 4: $75-80$

16. Jha N, et al. Prevalence of Adverse Drug reactions with commonly prescribed drug in different hospitals of Kathmandu valley. Kathmandu University Medical Journal. 2007;5:504-510

17. Tejal KG, et al. Patient safety adverse drug events in ambulatory care. The New England Journal of Medicine. 2003;348:1556-1564

18. Bilal Ahmed et al. Effects of Polypharmacy on Adverse Drug Reactions among Geriatric Outpatients at a Tertiary Care Hospital in Karachi: A Prospective Cohort Study . PLoS ONE .2014; 9(11):e112133

19. Rogie Royce Carandang et al. Knowledge and Attitudes on Adverse Drug Reaction Reporting of Selected Hospital-based Health Practitioners in Manila, Philippines.Scholars Academic Journal of Pharmacy (SAJP) .2015; 4(6): 301-307

20. Dr.Mohammed Abuzar Ghufran et al.A prospective observational study on monitoring,evaluating and reporting of ADRs in a tertiary care hospital .World Journal of Pharmacy and Pharmaceutical Sciences.2016; 5(2):736-752

21. Jamuna Rani.R. Priya.M. Hospitalization Caused by Adverse Drug Reactions(ADRs) in a Tertiary care Hospital in South India: A Retrospective Study .National Journal of Basic Medical Sciences .2011; 3(3) :227-230

22. Arulmani R, Rajendran S.D, Suresh B. Adverse drug reaction monitoring in secondary care hospital in south India. Br J Clin Pharmacol. 2007; 65(2): 210-216

23. Davies EC, Green CF, Taylor S, Williamson PR, Mottram DR: PLoS ONE .2009; 4(2): e443

24. Fadare JO, Agboola SM, Opeke OA, Alabi RA. Prescription patternand prevalence of potentially inappropriate medications among elderly patients in a Nigerian rural tertiary hospital. Ther Clin Risk Manag. 2013;6:115-20 
25. Lohani SP, Thapa P, Aryal UR, Satyal KR. Polypharmacy and geriatric patients: Patterns of prescribing in the Tribhuvan University Teaching Hospital in Nepal. J Nepal Health Res Council .2006;4(1)

26. Palanisamy S, et al. a study of assessment, monitoring, documentation and reporting of adverse drug reaction at a multi-specialty tertiary care teaching care hospital in south India. International Journal of Pharmatech Research. 2009;1:15191522

27. Sharma H, Aqil M, Imam F, Alam MS, Kapur P, Pillai KK. A pharmacovigilance study in the department of medicine of a university teaching hospital. Pharmacy practice. 2007;5(1):46-9

28. Michael A.Steiman et al. Do geriatric condition increase risk of ADR in ambulatory elders?Results from the VA GEM Drugs Study.J.Gerontol A BioSci Med Sci2011;66(4) : 444-451.

29. Marie-Laure Laroche et al.Is Appropriate medication use a major cause of adverse drug reactions in the elderly.Br J Clin Pharmacol.2007;63(2) :177-186

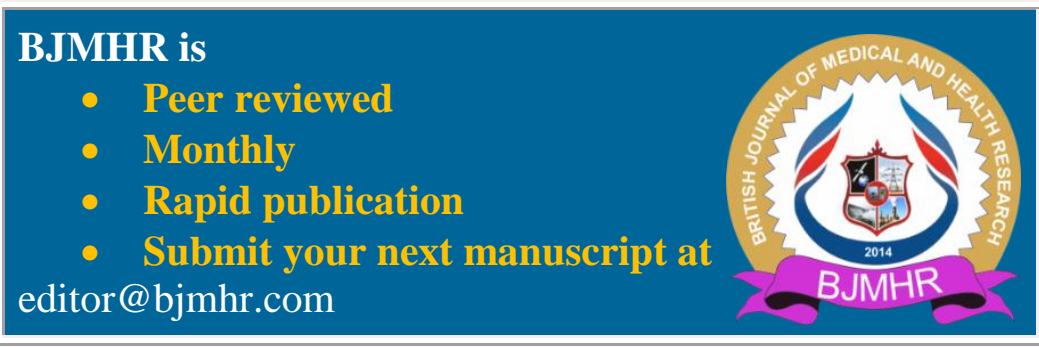

